\title{
Aspergillus aortitis: a case with near-complete occlusion of the brachial artery and large ascending aortic mycotic pseudoaneurysm in an immunocompetent patient
}

\begin{abstract}
Invasive cardiac Aspergillosis is an extremely rare life-threatening disease in immunocompetent hosts that has been described in the literature in a few cases. Most cases have presented with an underlying pulmonary Aspergillosis in the setting of an immuno compromised status. This is to report an exceptionally rare Aspergillus aortitis in an immunocompetent patient presenting with near-complete occlusion of the brachial artery and a large ascending aortic mycotic pseudoaneurysm. To our knowledge this is the first reported case of Aspergillus aortitis in an immunocompetent host presenting initially as intra arterial thrombosis without intracardiac thrombosis or valvular vegetation. The patient did not have any pre-existing pulmonary or mediastinal disease, but had a history of coronary artery bypass grafting 5 years prior to the infection.
\end{abstract}

Keywords: Aspergillus, aortitis, immunocompetent, thrombosis, cardiac surgery
Volume 3 Issue 4 - 2016

\author{
Rabab Elmezayen, Dima Youssef \\ Department of Internal Medicine, East Tennessee State \\ University, USA
}

\begin{abstract}
Correspondence: Dima Youssef, Associate professor, Department of Internal Medicine, Division of Infectious Diseases, East Tennessee State University, Box 70622,VAMC Bldg. I, Dogwood Ave, Johnson City, TN 376|4, USA, Tel + I 423 439 6380, Fax + 423439 7010, Email estecina@hotmail.com
\end{abstract}

Received: September 30, 2016 | Published: December 29 2016

\section{Introduction}

Aspergillus is a saprophytic, spore-forming ubiquitous fungus. Aspergillus exists in soil, water and decaying vegetation. Inhalation of the airborne conidia causes a multitude of diseases, ranging from asymptomatic colonization to disseminated infection. ${ }^{1}$ Invasive Aspergillosis is an opportunistic infection that mostly affects immunocompromised patients. Invasive Aspergillosis can occur virtually in any organ. Aspergillus infection of the ascending aorta after cardiopulmonary bypass surgery has rarely been reported. Despite antifungal therapy and surgical intervention, Aspergillus aortitis can be fatal and carries a poor prognosis.

Most reported cases of Aspergillus aortitis were described in patients who underwent some sort of cardiothoracic surgery. We report a case of Aspergillus brachial artery thrombosis with nearcomplete occlusion and vascular compromise to the limb requiring urgent thrombectomy. Fungal organisms identified at the thrombus were morphologically consistent with Aspergillus species. This has lead to the discovery of an ascending aortic pseudoaneurysm, and thus the diagnosis of Aspergillusn aortitis. There was no aortic valve vegetation.

\section{Case presentation}

A 57-year-old white male patient with known history of coronary artery disease, morbid obesity, obstructive sleep apnea, hypertension, hyperlipidemia, atrial fibrillation, and tobacco use, presented to a local emergency room with a sudden sharp right arm pain, followed a few hours later by numbness of the right hand. His physical exam was normal except for a very weak pulsation of the right brachial artery. His initial workup included a normal chest $\mathrm{x}$-ray with no acute cardiopulmonary process. A doppler ultrasound of the right upper extremity indicated thrombosis with near-complete occlusion of the distal right brachial artery. He was urgently seen by vascular surgery and underwent thrombectomy of the distal right brachial artery thrombus that was sent for pathology. Hematoxylin Eosin stain and Grocott's methenamine silver stain showed fungal organisms identified at the thrombus, that had thin separate hyphae with regular branching and that were morphologically consistent with Aspergillus species (Figures 1-6). Subsequently, he was started on intravenous voriconazole, after his EKG, liver function tests were evaluated revealing a normal QTc interval, and liver enzymes respectively. Patient was then evaluated for evidence of disseminated Aspergillosis. His blood cultures have been negative. Aspergillus galactomannan antigen was negative, and his HIV $1 / 2$ Antibody screen was negative. A computed tomography (CT) scan of the chest with angiography showed an ascending aortic pseudoaneurysm with large soft tissue component, representing a mycotic aneurysm. The contrast opacified lumen of the pseudoaneurysm measured $2.8 \times 3.8 \mathrm{~cm}$ and there was a portion of the pseudoaneurysm more superiorly that was thrombosed measuring $6.3 \times 7.2 \mathrm{~cm}$ (Figures $7 \& 8$ ). His lungs were clear without evidence of pulmonary nodules or consolidation. CT scan of the brain with contrast and a complete ophthalmological examination were unremarkable. Transthoracic and transesophageal echocardiograms showed no major cardiac finding and no evidence of valvular vegetations. He was then transferred to a tertiary care center where he underwent a homograft of cryopreserved human tissue given the fact that he has an infected mediastinum. He was continued on Voriconazole orally, and he will receive it for life.To note that, five years prior to this event, he underwent an urgent coronary artery bypass grafting $(\mathrm{CABG}) \mathrm{X} 3$ with reversed autogenous saphenous vein graft from the aorta to the left anterior descending artery, from the aorta to the ramus intermediate, from the aorta to the obtuse marginal artery, along with endoscopic vein harvest left and right greater saphenous veins (from knee to groin bilaterally). 

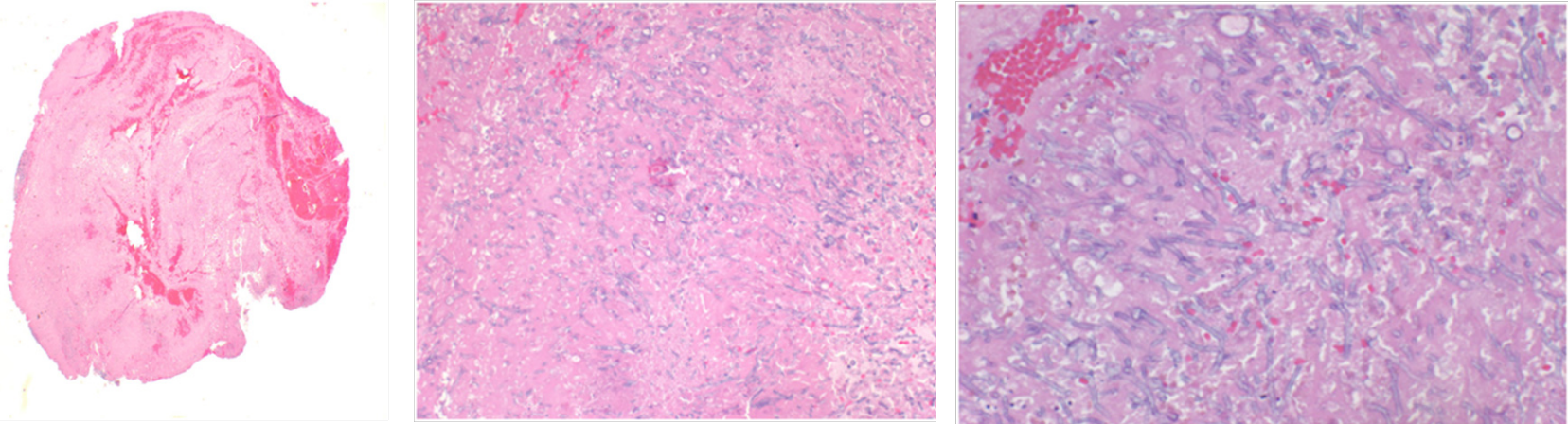

Figures I-3 Hematoxylin-Eosin stain showing thin septate hyphae with regular branching.
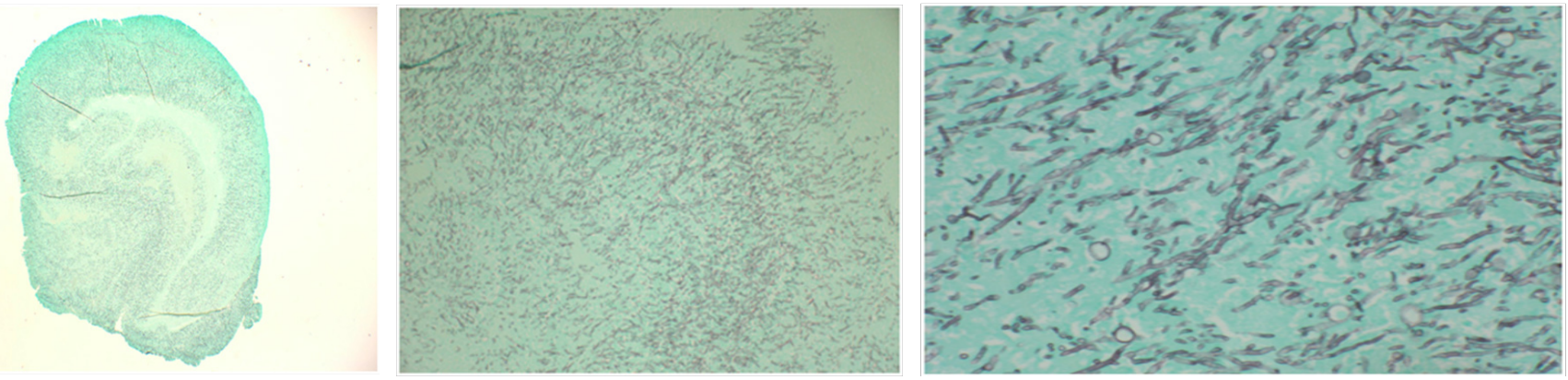

Figures 4-6 GMS stain showing the same findings.

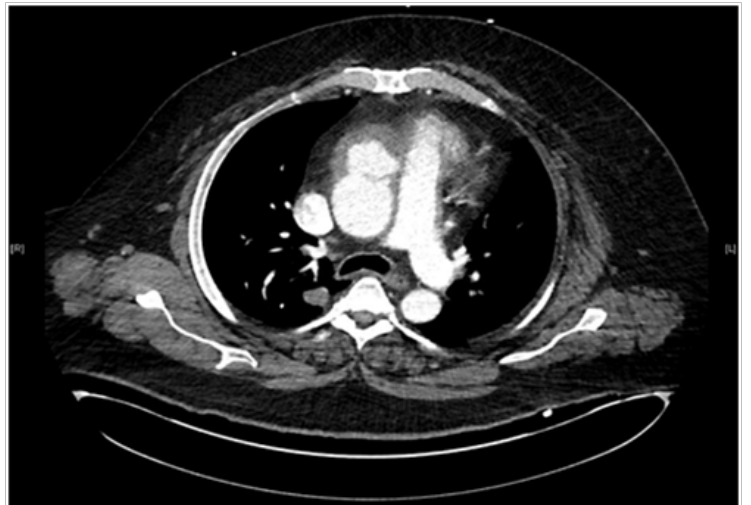

Figure 7 Contrast opacified lumen of the pseudoaneurysm measuring $2.8 \times 3.8 \mathrm{~cm}$.

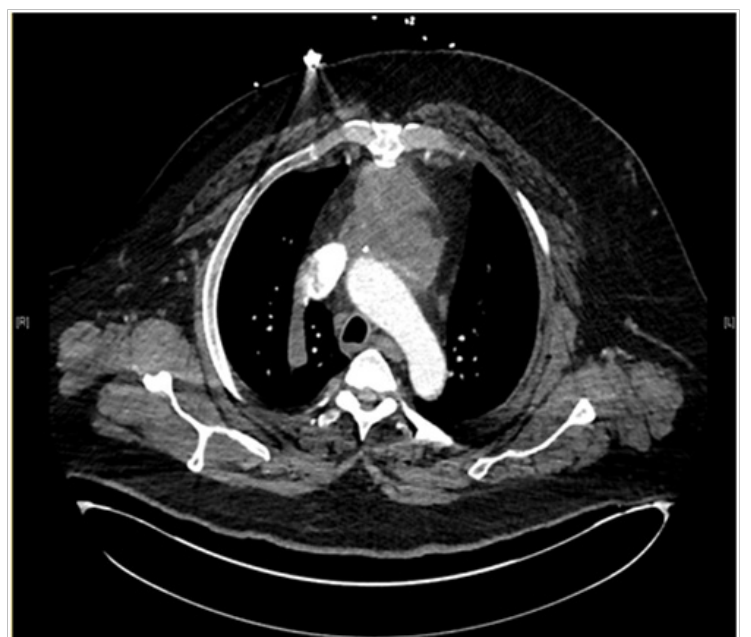

Figure $8 \mathrm{~A}$ portion of the pseudoaneurysm located superiorly in mediastinum is thrombosed and measures $6.3 \times 7.2 \mathrm{~cm}$.

\section{Discussion}

Our patient represents one of the rare cases of invasive Aspergillosis in the setting of a history of previous CABG 5years prior to this infection. Fungal aortitis remains a rare disease occurring mostly in patients with predisposing host conditions. Aspergillus aortitis is an uncommon infection with high mortality and has been reported in several cases in the literature following surgical procedures, mainly cardiopulmonary bypass. While invasive Aspergillosis occurs typically in severely immuno compromised patients, cases of surgical site infection have been reported in immunocompetent individuals. Underlying immunosuppressive disorder is not necessary. The source is presumed to be airborne infection during the surgical procedure. Special care of the ventilation system in the operating room is necessary to prevent such infection. ${ }^{2,3}$ The clinical presentation is usually with septic symptoms including fever, night sweats and lethargy. This patient did not present with the classical symptoms, but rather was asymptomatic until symptoms of ischemia to the right upper extremity started secondary to arterial embolism. He also did not have any respiratory symptoms or skin lesions. He did not have a prolonged hospitalization. He has no history of intravenous devices or intravenous drug use.

The diagnosis of Aspergillus aortitis is difficult because of the localization of the infection and the absence of endocarditis vegetation. ${ }^{2}$ Transesophageal echocardiography proved very useful in the diagnosis of aortitis. ${ }^{4,5}$ Computed tomography scan, magnetic resonance imaging and angiography might be more helpful. The role of antibody tests and molecular tests of blood samples has not been established yet.

A search of the literature for post operative Aspergillosis occurring after surgical procedures that were unrelated to disseminated infection or colonization by Aspergillus found that out of 500 patients with postoperative Aspergillosis, 188 had an infection following heart surgery. ${ }^{2}$ Males were more commonly affected (around $70 \%$ ), and the aortic 
valve was the mostly involved (60.5\%). Infection may occur from weeks to several years after surgery. Aspergillus fumigatus was mostly isolated $(58.7 \%)$ on fungal cultures of the infected tissues, ${ }^{2}$ while blood cultures remain almost always negative. Aspergillus is a rare cause of aortic graft infection. ${ }^{3}$ As such, the first case of Aspergillus aortitis in an immunocompetent individual was diagnosed immediately after percutaneous coronary intervention to an aortocoronary bypass graft. ${ }^{4}$

Aspergillus aortitis has a propensity for late embolization. The first report of fatal postoperative Aspergillus infection in an aortic pseudoaneurysm, with arterial embolization to the brain occurred threemonths after successful coronary bypass surgery. ${ }^{6}$ Similarly, a case of Aspergillus niger aortitis presented as lower limb embolisms 9months after CABG. ${ }^{7}$ Likewise, acute aortic occlusion from a saddle embolus originating from Aspergillus niger aortitis happened 4 months after aortic valve replacement. ${ }^{8}$ Another immunocompetent patient had embolization to the cerebral artery and cubital artery three years following CABG. It was only on necropsy that parietal aortic Aspergillosis with septic embolisms in the brain, kidney, liver, fingers was diagnosed. ${ }^{9}$

Like the case of our patient, aortic pseudoaneurysm may develop. ${ }^{3,10}$ An immunosuppressed patient had heart transplantation 4months prior to the diagnosis of a large pseudoaneurysm of the ascending aorta. He underwent surgical repair, and the cultures were positive for Aspergillus fumigatus. ${ }^{11}$

Aspergillus aortitis can occur following aortic valve replacement. This was reported in a patient that died 18 days following combined aortic valve replacement. Ascending aortic perforation with multiple fungal lesions due to invasion of the aortic wall by Aspergillusfumigatus was noted. ${ }^{12}$ Another case of Aspergillus aortitis occurred after aortic valve replacement for native aortic valve aspergillar endocarditis. This latter developed 18 months after coronary artery bypass surgery. Despite aortic valve replacement and amphotericin B therapy, the patient died of Aspergillus species aortitis with occlusion of the coronary ostia and bypass grafts. ${ }^{13}$

Treatment of Aspergillus aortitis requires the combination of antifungal therapy and surgical intervention. ${ }^{2}$ This patient underwent thrombectomy of the distal right brachial artery thrombus and surgical resection and grafting of aortic mycotic aneurysm. He was treated with intravenous voriconazole before and at the time of the surgery, and then switched to the oral form of the medication. He is supposed to be on it for life. It has been reported that voriconazole with or without liposomal amphotericin B formulation is regarded as the medical treatment of choice in most cases of invasive aspergillus infections. ${ }^{14}$ Our patient responded well to voriconazole monotherapy with no major side effects. Voriconazole has been related to improved survival, less nephrotoxicity, and electrolyte imbalance, and less infusion related events. However, it may be associated with bony changes with fluorinated deposits as a possible side effect, and may cause prolonged QT interval in patients with already established heart disease.

Overall, there is a low ante-mortem diagnosis (43.5\%), usually following surgical intervention, and the mortality is high up to $>80 \%$ despite intervention. ${ }^{2}$ This could be due to a delayed diagnosis, that allows the fungus to establish local necrosis, pseudoaneurysm formation, and embolization to large arteries. Our patient has a unique presentation with arterial thrombosis and aortic mycotic aneurysm requiring surgical treatment in combination with the antifungal therapy. His immunocompetent status improved his survival and increased his chance of better response to the treatment.

\section{Conclusion}

Our case demonstrates that Aspergillusaortitis may occur in immunocompetent previously healthy individual following cardiac intervention and can be fatal. The scarcity of fungal aortitis demands knowledge of the group of at-risk patients. Our case is a reminder that we should have a high index of suspicion in patients with unusual presentation of arterial thrombosis without history or risk factors for hypercoagulable state. Aspergillus aortitis is typically found after aortic valve or coronary surgery. It commonly leads to lethal multiorgan dissemination without involvement of the intracardiac structure. This entity should be considered in patients with persistent fever and negative blood cultures after open-heart surgery involving significant aortic wall damage, regardless of the postoperative period. Survival of the patient with Aspergillus aortitis is dependent on early initiation of aggressive medical and surgical treatment. Successful treatment requires rapid diagnosis, surgical debridement in conjunction with long term antifungal therapy, often with voriconazole. In order to improve the outcome, better diagnostic methods are needed. Delayed surgical intervention and medical therapy alone are associated with high mortality rates. Special attention to the ventilation systems in operating rooms should be made.

\section{Acknowledgements}

None.

\section{Conflict of interest}

The author declares no conflict of interest.

\section{References}

1. Soubani AO, Chandrasekar PH. The clinical spectrum of pulmonary Aspergillosis. Chest. 2002;121(6):1988-1999.

2. Pasqualotto AC, Denning DW. Post-operative Aspergillosis. Clin Microbiol Infect. 2006;12(11):1060-1076.

3. Aguado JM, Valle R, Arjona R, et al. Aortic bypass graft infection due to Aspergillus: report of a case and review. Clin Infect Dis. 1992;14(4):916921

4. Khumri TM, Joslin NB, Nayyar S, et al. Transesophageal echocardiographic diagnosis of Aspergillus fumigatus aortitis after percutaneous coronary intervention. $J$ Am Soc Echocardiogr. 2006;19(8):1072.e9-1072.e11.

5. Duygu H, Nalbantgil S, Ozerkan F, et al. Aspergillus niger aortitis after aortic valve replacement diagnosed by transesophageal echocardiography. Echocardiography. 2006;23(5):405-406.

6. Gray R, Kaplan L, Matloff J, et al. Aortic pseudo-aneurysm with Aspergillus aortitis. An unusual complication of coronary bypass surgery. Chest. 1986;89(2):306-308.

7. Noordally SO, Sohawon S, De Bels D, et al. Late onset of Aspergillus aortitis presenting as femoral artery embolism following coronary artery bypass graft surgery. Acta Medica (Hradec Kralove). 2011;54(4):175176.

8. Jamieson RW, Wallace WA, Din JN, et al. Acute aortic occlusion with sudden paraplegia secondary to Aspergillus niger embolism from Aspergillus niger aortitis. J Vasc Surg. 2011;54(5):1472-1474.

9. Abenza Abildua MJ, Fuentes Gimeno B, Morales Bastos C, et al. Stroke due to septic embolism resulting from Aspergillus aortitis in an immunocompetent patient. J Neurol Sci. 2009;284(1-2):209-210.

10. Sanchez Recalde A, Maté I, Merino JL, et al. Aspergillus aortitis after cardiac surgery. J Am Coll Cardiol. 2003;41(1):152-156. 
11. Ronco F, Simsir S, Czer L, et al. Incidental finding by two-dimensional echocardiography of a mycotic pseudoaneurysm of the ascending aorta after orthotopic heart transplantation. J Am Soc Echocardiogr. 2010;23(5):580.e1-580.e3.

12. Hanvesakul R, Deshpande R, Carr CS, et al. Aspergillus aortitis: a cause for aortic perforation in a patient following combined aortic valve surgery and liver transplantation. Interact Cardiovasc Thorac Surg 2004;3(4):544-546.
13. Opal SM, Reller LB, Harrington G, et al. Aspergillus clavatus endocarditis involving a normal aortic valve following coronary artery surgery. Rev Infect Dis. 1986;8(5):781-785.

14. Herbrecht R, Denning DW, Patterson TF, et al. Invasive fungal infections group of the european organization for research and treatment of cancer and the global aspergillus study group. Voriconazole versus amphotericin B for primary therapy of invasive Aspergillosis. $N$ Engl J Med. 2002;347(6):408-415. 